\title{
The Effect of a Variable Anisotropic Continuum Source upon the Broad Emission-Line Profiles and Responses
}

\author{
Mike Goad \\ Space Telescope Science Institute, 3700 San Martin Drive, Baltimore, \\ $M D 21218, U S A$ \\ Ignaz Wanders \\ Dept. of Astronomy, Ohio State University, 174 West 18th Avenue, \\ Columbus, OH 43210, USA
}

\section{Introduction}

Optical monitoring data over several years in combination with the reconstructed 1-d response function for $\mathrm{C}$ IV obtained from the $1993 \mathrm{HST}$ monitoring campaign of NGC 5548, reveal that: (a) radial motion does not dominate the gas kinematics (Korista et al. 1995); (b) line-profile variations occur in fixed regions of velocity space, a core and two wing components, are stochastic in nature, and appear to be unrelated to reverberation effects (Wanders \& Peterson 1996). Wanders et al. (1995) showed that these variations are broadly consistent with a spherical BLR geometry populated with optically thick clouds following randomly inclined circular Keplerian orbits, and illuminated by an anisotropically emitting point source of ionizing continuum radiation. Here we provide a brief description of this model and summarize its basic properties. A more thorough analysis is presented in Goad \& Wanders (1996).

\section{An Anisotropically Illuminated BLR Model}

For simplicity we model the continuum source as an isotropic component with constant strength $F_{i}$, in combination with a bi-conical component of constant strength $F_{b}$, semi-opening angle $\omega$, and line of sight inclination $i$. Thus clouds inside the beam receive a total flux $F_{i}+F_{b}$, whilst those outside the beam receive $F_{i}$ only. Figure 1 shows model broad emission-line profiles for a geometrically thick spherical BLR, with inner and outer radii of 1 and 10 lt-days respectively, power-law emissivity distribution $\varepsilon(r) \propto r^{-2}$, differential covering factor $d C(r) \propto r^{0} d r$, and beam strength $R=F_{i} / F_{b}=1 / 3$. The velocity at the inner radius $V\left(R_{i n}\right)=2 \times 10^{4} \mathrm{~km} \mathrm{~s}^{-1}$. Clearly, just by varying $i$ and $\omega$ a large fraction of the observed range in profile shapes can be readily reproduced.

The proposed model displays a number of interesting properties. First, the detection of single peaks is strongly favored. Secondly, double-peaked profiles have larger FWHM and larger EWs than single-peaked profiles. Finally, doublepeaked profiles can still be observed even if one beam is obscured.

Observationally, the so called 'disk-like emitters' (Eracleous \& Halpern 1994) display stronger FWHM and larger EW than single-peaked profiles. Fur- 


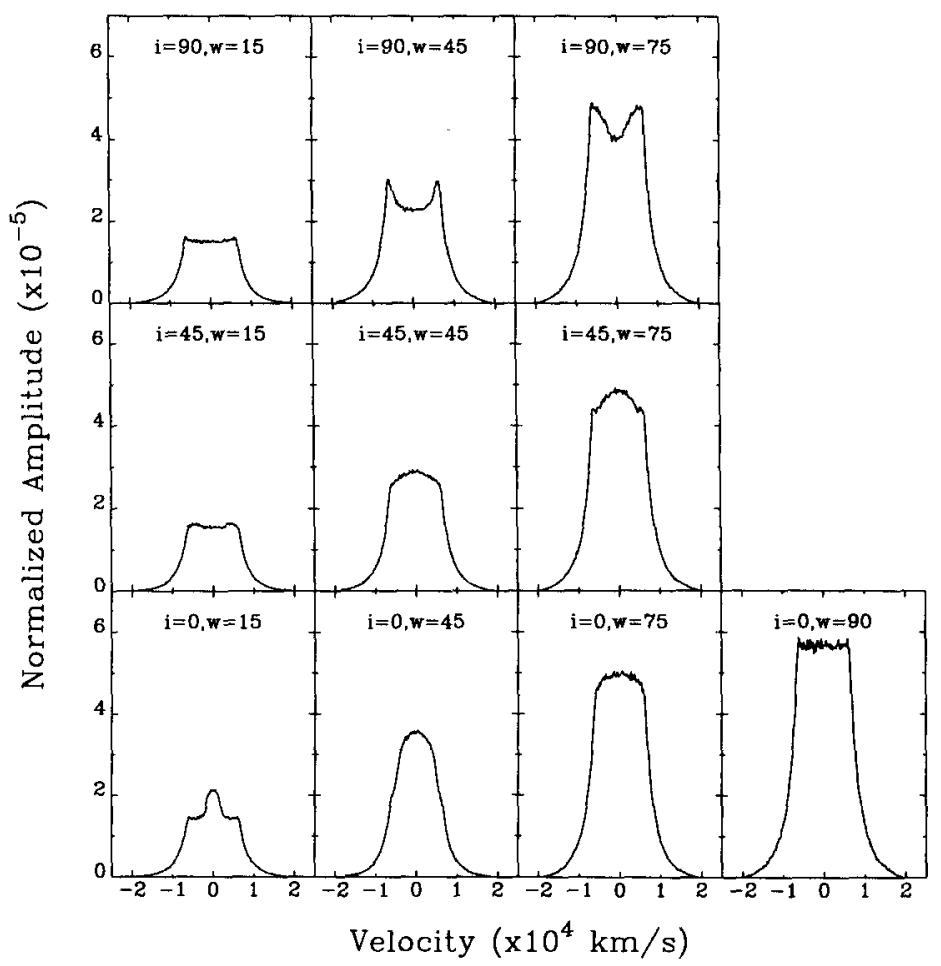

Figure 1. Normalized broad emission-line profiles for an anisotropically illuminated BLR for a range of $i$ and $\omega$.

thermore, the double-peaked Balmer lines and single-peaked UV lines observed in Arp 102B can be modeled by assuming a strong variation in the spectral energy distribution of the ionizing continuum source with cloud elevation. Finally, the line asymmetries and displaced single peaks observed in some objects, can here be accommodated by invoking small cloud numbers.

Acknowledgments. M.R. G. and I.W. gratefully acknowledge financial support through NASA grants NAG5-2111 and NAG5-2477, respectively.

\section{References}

Eracleous, M., \& Halpern, J.P. 1994, ApJS, 90, 1.

Goad, M.R., \& Wanders, I. 1996, ApJ, in press.

Korista, K.T., et al. 1995, ApJS, 97, 285.

Wanders, I., et al. 1995, ApJ, 453, L87.

Wanders, I., \& Peterson, B. M. 1996, ApJ, 466, 174. 